\title{
Spiral Shocks in Accretion Disks with SPH
}

\author{
James Rhys Murray \\ Canadian Institute for Theoretical Astrophysics, University of Toronto, \\ Ontario, M5S 3H8, Canada
}

\begin{abstract}
Smoothed Particle Hydrodynamics (SPH) is now seen as a numerical scheme well suited to the study of accretion disks. SPH simulations have been conducted of cataclysmic variable disks (Lubow 1991, Murray 1996, Armitage and Livio 1996), galactic disks (Artymowicz and Lubow 1989), and protostellar disks (Artymowicz and Lubow 1994). It is therefore important to test the technique against theory and other numerical results to obtain an estimate of the accuracy and reliability of SPH in this context. Previously SPH has been tested against standard stationary and time-dependent results of viscous thin disk theory (Murray 1996). Strictly these tests relate to disks where 'viscous' terms dominate pressure terms in the equations of motion.

In this paper we describe tests of the code more appropriate for hot disks where pressure forces are relatively more important than viscosity. Specifically we consider the form of the spiral density waves that can be excited in a disk by a perturbing gravitational potential. Very low mass perturbing bodies excite linear spiral waves which redistribute angular momentum in the disk. For increasingly massive perturbers, the disk response becomes nonlinear and eventually shocks form. In the standard formulation of SPH, an artificial viscosity term is added to the SPH equations to improve shock capture. This is equivalent to introducing a fixed ratio of shear to bulk viscosity into the equations of motion. In Eulerian schemes, artificial viscosity has been discarded in favour of other more accurate, less dissipative schemes for resolving shocks. The continued use of artificial viscosity in SPH has become a source of 'friction' between numericists. The simulations described here demonstrate the scheme's ability to resolve spiral shocks, and show that SPH is a valuable tool for probing the structure of tidally perturbed accretion disks.
\end{abstract}

\section{References}

Armitage, P. J., Livio, M. 1996, ApJ, accepted

Artymowicz, P., Lubow, S.H. 1989, in Dynamics of Astrophysical Discs, CUP, 1989, 211

Artymowicz, P., Lubow, S.H. 1994, ApJ, 421, 651

Lubow, S. H. 1991, ApJ, 381, 268

Murray, J. R. 1996, MNRAS, 279, 402 